\title{
Thoughts on Standards Materials and Analytical Routines for Electron Backscatter Diffraction (EBSD)
}

\author{
Matthew M. Nowell ${ }^{1}$, and Stuart I. Wright ${ }^{1}$ \\ ${ }^{1 .}$ EDAX, A Division of Ametek, Materials Analysis Division, Draper UT USA
}

Since the development and subsequent commercialization of automated analysis of Electron Backscatter Diffraction (EBSD) patterns for orientation mapping [1], the development of standards for EBSD within the microanalysis community has not kept pace with the adoption rate of the technique in general. When compared with Energy Dispersive Spectroscopy (EDS), where numerous standard materials and analytical routines are easily available to help evaluate the performance of an EDS system, there are few established benchmarks for EBSD users [2,3]. The goal of this paper is to present the materials and methods used at EDAX to evaluate and demonstrate EBSD system performance.

For EBSD, sample preparation is a key factor in obtaining quality results [4]. Inconel 600, a nickelbased superalloy, is a material that is generally easy to prepare for EBSD, and has a relatively large backscatter coefficient for stronger EBSD pattern intensity. It also has a benign room-temperature oxidation behavior that allows for constant pattern quality from a prepared sample over time. The polycrystalline microstructure allows characterization of grain size and shape, and the presence of twin boundaries within the material provides a reference misorientation boundaries that are easily available. Though often considered nominally recrystallized, often some amount of plastic deformation can be detected within the grains. These factors make this material an ideal candidate for a standard EBSD mapping material.

Single crystal silicon is also a useful standard material for EBSD. With a crystal of known orientation, it is straightforward to determine if the correct orientation is being calculated. It is also useful to determine the precision of EBSD measurements, by mapping an area of single crystal and measuring the spread in the determined orientations [5]. However enough oxidation can occur with silicon at roomtemperature to degrade EBSD pattern quality and subsequent orientation measurements. Care must be used to remove this oxide layer for best results.

Beyond materials, there are also analytical metrics used to describe EBSD system performance. Indexing success rate (ISR) is one used to describe the percentage of acquired EBSD pattern analyzed correctly [4]. However, this metric can be difficult for true comparisons for multiple reasons: there are different approaches to defining indexing success, different areas on a given sample may produce different results, mapping the same area may result in EBSD pattern degradation from contamination effects, and EBSD detector settings can influence performance. One approach for a solution is to generate a set of simulated EBSD patterns with a known orientation at each point that can then be analyzed and the resulting measurements compared with the known orientations on a point-by-point basis [6]. This approach disassociates the effects of the detector system from the effects of the pattern indexing algorithm.

One approach for evaluating EBSD detector performance is characterizing the noise in the EBSD background image. Generally, the background is obtained by rastering the electron beam over enough grains that the local orientation information is averaged out and the remaining characteristic signal 
representing the non-diffracted, backscattered signal onto the EBSD detector is obtained. Typically, this background is either subtracted or divided from an individual EBSD pattern to flat-field the image intensity and enhance the contrast for improved band detection. For noise characterization, two background images can be captured and subsequently subtracted one from the other. The standard deviation of the resultant difference image matrix provides a measure of detector noise at typical EBSD imaging conditions and can be used in subsequent signal-to-noise calculations. However, this noise measurement does not directly correlate with EBSD pattern quality, as shown in Figure 1. Here is EBSD background noise level is plotted for two Inconel 600 samples: one polished for 3 hours with $0.05 \mu \mathrm{m}$ colloidal silica (labeled CS) and one polished for 10 minutes with $0.3 \mu \mathrm{m} \mathrm{Al}_{2} \mathrm{O}_{3}$ (labeled A12O3). These curves are essentially identical, even though the EBSD pattern quality is quite different. Image quality, an EBSD pattern analysis metric that can identify the quality of a diffraction image, is also shown in Figure 1 for the two preparation conditions [7]. The IQ measurements complements the noise measurement metric, and differentiates the sample preparation effects on the final EBSD pattern.

References:

[1] B.L. Adams, S.I. Wright, and K. Kunze, Metallurgical Transactions A 24 (1993), p.819.

[2] T.B. Britton, et. al., Ultramicroscopy 135 (2013), p. 126.

[3] M.D. Vaudin, et. al., Ultramicroscopy 148 (2015), p. 94.

[4] M.M. Nowell, R.A. Witt, and B. True, Microscopy and Microanalysis 11 S02 (2005), p.504.

[5] S. I. Wright, M. M. Nowell, and J.A. Basinger, Microscopy and Microanalysis 17 S02 (2011), p.406.

[6] F. Ram, S. Singh, S.I. Wright, and M. De Graef, Ultramicroscopy Submitted (2016).

[7] S.I. Wright and M.M. Nowell, Microscopy and Microanalysis 12 (2006), p.72.

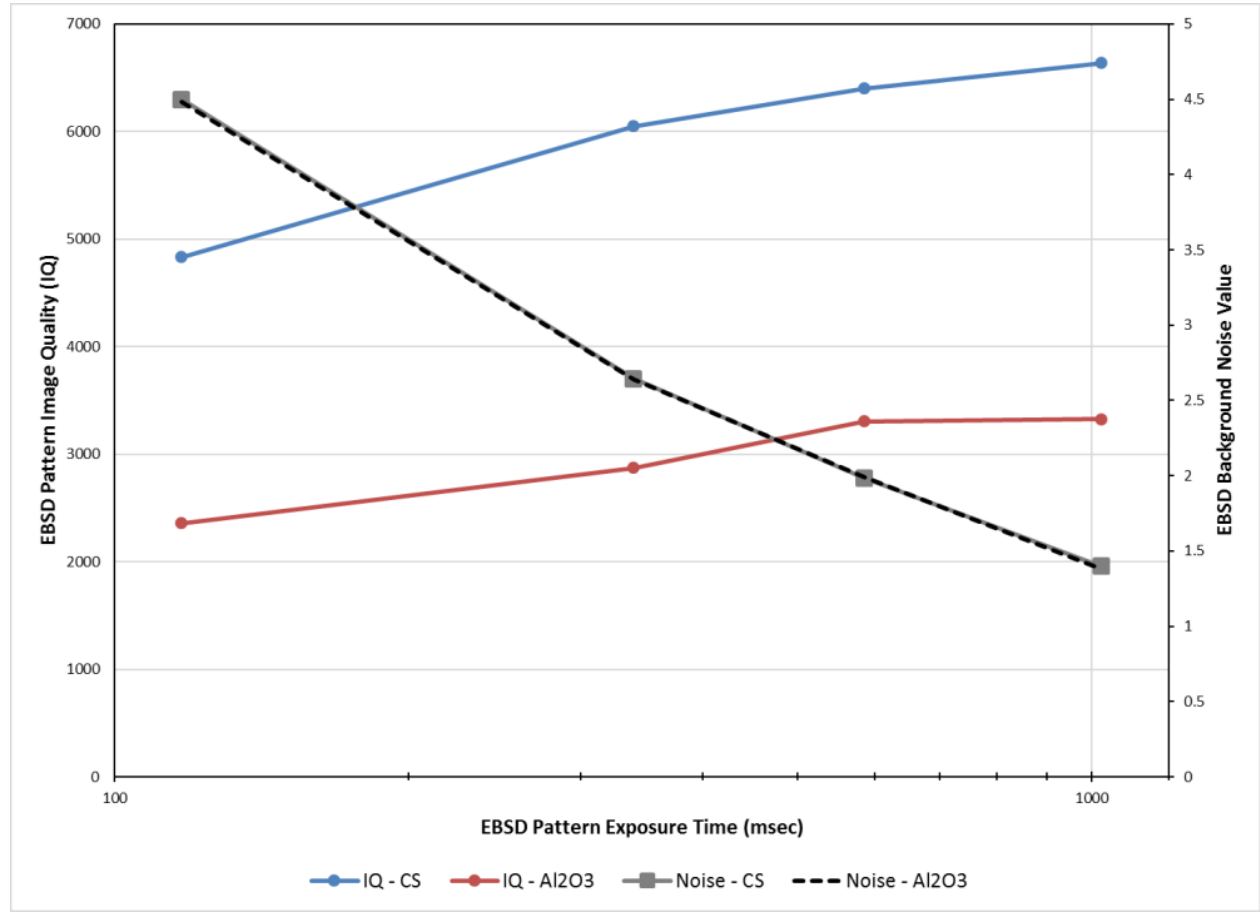

Figure 1. EBSD pattern Image Quality (IQ) and EBSD background noise distributions for Inconel 600 sample polished down to $0.3 \mu \mathrm{m} \mathrm{Al}_{2} \mathrm{O}_{3}$ and to $0.05 \mu \mathrm{m}$ colloidal silica. 